\title{
L'autre côté de la croissance de l'emploi en Espagne : une précarité qui se perpétue
}

La otra cara del crecimento del empleo en España : una precariedad que no cesa The other face of growth in spanish employment : a continuing precarity

\section{Fausto Miguélez et Carlos Prieto}

\section{(2) OpenEdition}

\section{Journals}

Édition électronique

URL : http://journals.openedition.org/travailemploi/4221

DOI : 10.4000/travailemploi.4221

ISSN : 1775-416X

Éditeur

DARES - Ministère du Travail

Édition imprimée

Date de publication : 15 septembre 2008

Pagination : 45-57

ISSN : 0224-4365

\section{Référence électronique}

Fausto Miguélez et Carlos Prieto, «L'autre côté de la croissance de l'emploi en Espagne : une précarité qui se perpétue », Travail et Emploi [En ligne], 115 | juillet-septembre 2008, mis en ligne le 18 février 2011, consulté le 04 mai 2019. URL : http://journals.openedition.org/travailemploi/4221 ; DOI : 10.4000/travailemploi.4221 


\title{
L'autre côté de la croissance de l'emploi en Espagne: une précarité qui se perpétue
}

\author{
Fausto Miguélez (*), Carlos Prieto (**)
}

Les experts s'accordent à dire que le marché du travail espagnol, par rapport à celui d'autres pays à l'économie avancée, se caractérise par une précarité particulière, qui touche une frange importante des salariés et se manifeste par un taux élevé de contrats précaires. Tous les chercheurs et les acteurs sociaux en Espagne (1) pointent ce phénomène, et certaines voix au-delà de nos frontières en sont venues à parler de l'Espagne comme de la «championne de l'emploi précaire» (Liaisons Sociales Magazine, 2000). Cet article tente de comprendre ce qui fait la spécificité de la précarité propre au marché du travail espagnol.

Nous commencerons par définir ce que nous entendons par «précarité de l'emploi». Pour cela, nous montrerons tout d'abord comment, au cours des dernières décennies, s'est produit en Espagne un profond changement de la norme sociale de l'emploi, qui a ouvert la voie à la normalisation de formes d'emploi «temporaires» et qui a été suivi par une très forte croissance des contrats dits temporaires. Par contrats «temporaires», on comprend en Espagne toute sorte de contrats de travail dont la durée est établie comme limitée, y compris les contrats intérimaires. Il y en a une grande diversité: contrats «d'œuvre ou service», «éventuels (en raison d'une augmentation de la demande de production dans l'entreprise, «intérimaires », «en formation» et «en stage professionnel» (voir le Estatuto de los Trabajadores). Tous ces contrats sont justifiés par des «raisons objectives»: leur courte durée est indiquée par l'objet même du contrat. De 1984 à 1994 fut mis en vigueur un contrat à caractère temporaire, limité dans sa durée (entre trois mois et trois ans), qui ne demandait aux entreprises

(*) Professeur de sociologie à l'université autonome de Barcelone (UAB) et directeur du QUIT; fausto.miguelez@uab.es

(**) Professeur de sociologie à l'université Complutense de Madrid; cprieto@cps.ucm.es

(1) Un mémoire du Conseil économique et social espagnol sur l'Espagne en 2005 parle «du nombre excessif de contrats "temporaires" qui soutient le marché du travail» espagnol (CES, 2006: 239). aucune justification objective: il fut mis en place par le gouvernement «comme mesure de promotion de l'emploi », justification qui était incorporée à sa dénomination (Contrato temporal como medida de fomento del empleo). Il s'agit donc d'une dénomination en négatif: sont temporaires tous les contrats de travail qui ne sont pas des contrats à durée indéterminée (Non Permanent Contracts dans la terminologie anglaise de la Commission européenne)(2).

Nous insisterons ensuite sur la multidimensionalité de ce type d'empois pour ce qui concerne les conditions d'emploi, ainsi que sur la profondeur de leur pénétration sur le marché du travail espagnol et leur prolongement dans le temps. Nous conclurons en soulignant la spécificité du problème et de la définition de la précarité de l'emploi en Espagne.

\section{L'emploi précaire : de la définition "profane" à la définition "scientifique"}

Sans pouvoir faire le tour du long débat sur la définition de l' «emploi précaire» ou de la «précarité de l'emploi», il semble possible, en s'appuyant sur la littérature économique et sociologique internationale, d'arriver au moins à trois conclusions générales. Premièrement, la «précarité de l'emploi» est devenue un problème social qui, sous diverses dénominations, touche tous les pays à économie avancée, même si elle est considérée comme le prix à payer pour la flexibilité exigée aujourd'hui par une économie compétitive (CASTELS, 1999,

(2) Il faut souligner qu'Eurostat opère d'une façon semblable. L'agence européenne utilise le terme «emplois temporaires» dans le même sens qu'en Espagne. Le terme anglais est Temporary Contracts. Pour que cette particularité linguistique apparaisse clairement, on écrira ici le terme temporaire entre guillemets. 
volume 1; CARNOY, 2001)(3). Deuxièmement, cette «précarité»s'inscrit dans des cadres sociaux très différents, qui révèlent des réalités problématiques tout aussi différentes: qu'y a-t-il de commun, par exemple, entre un chômeur danois qui a droit à des allocations d'assurance-chômage pendant quatre ans à un taux de remplacement de $90 \%$ de son dernier salaire (MADSEN, 2004) et un chômeur espagnol qui, dans le meilleur des cas, aura droit à des allocations d'assurance-chômage pendant deux ans à un taux de remplacement de $70 \%$ de son dernier salaire ? Troisièmement, la façon dont cette diversité est pensée et interprétée (4) varie beaucoup d'un pays à l'autre. C'est aussi la conclusion à laquelle arrive Düll en commentant les différentes façons dont est perçu l'emploi précaire dans les pays qui ont participé au projet de recherche ESOPE (voir note 4): "One of the assessing precarious employment in a comparative perspective way is connected to the notion or 'precarious employment' itself and the different meaning it may take in the national labour market context. The perception of 'precarious employment' is imbedded in the ideological and political discourse of a country, its actual national regulatory and institutional context and its production model" (DülL, 2003, p. 3).

Si on accepte de dire, comme nous le faisons, que la «perception, et donc la définition, de la précarité de l'emploi est déployée (d'abord) dans le discours idéologique et politique du pays», il est cohérent de ne pas en élaborer une définition «scientifique» sans expliciter préalablement la définition «profane» déployée par ce «discours» en Espagne(5) Le discours sur la «précarité» de l'emploi en tant que problème social s'y est généralisé au moins depuis la fin des années 1980 (voir, par exemple, les articles publiés chez Miguélez, Prieto, 1991 et

(3) Le succès éditorial qu'a connu le livre de Sennett, The Corrosion of Character, dans tous ces pays, peut être interprété comme un signe de cette universalité.

(4) Pour une vision d'ensemble de ces différences, consulter l'article de TuChSzIRER (2005), où elle soutient que «le concept de précarité de l'emploi est loin de constituer, d'un pays à l'autre une catégorie homogène» (p. 23). D'une façon plus précise, on peut lire les documents présentés au séminaire international organisé par les chercheurs du projet ESOPE (European Study of Precarious Employment), intégré dans le European Framework 5 Programme sur "Risk and insecurity in flexibles economies" à l'université de Warwick le 23 et 24 mai de 2003. Les pays qui ont participé à - et ont été objet de - la recherche ont été la France, l'Allemagne, l'Italie, l'Espagne et le Royaume-Uni.

(5) L'idée de la convenance - ou nécessité - de tenir compte de la définition profane est présente chez d'autres auteurs. Voir par exemple Cingolani (2007:2): «On ne peut en la matière s'en tenir seulement à la dimension technique des spécialistes et des experts, mais il convient aussi de prendre en compte les passages et les circulations entre formes savantes et formes militantes dans un contexte historique visiblemement marqué par la revendication ouvrière et par une vie syndicale attentive aux transformations des rapports de production et de la condition des salariés ».
1999(6)) mais s'il a pris une telle prégnance, c'est surtout parce que la précarité est au cœur du discours revendicatif porté constamment et systématiquement par l'ensemble du syndicalisme espagnol et tout particulièrement, par les deux confédérations syndicales les plus reconnues et les plus représentatives, la Union General de Trabajadores (UGT) et la Confederation General de Comisiones Obreras (CCOO).

La définition syndicale de la précarité de l'emploi est caractérisée en Espagne par deux traits principaux (7). Tout d'abord, elle est devenue pour les syndicats l'expression métaphorique qui condense mieux que tout autre le moment actuel de la «question sociale». Pendant plusieurs années, le mot d'ordre revendicatif du syndicalisme espagnol au Premier Mai s'y est rapporté explicitement: "Por un empleo seguro, estable y con derechos (Pour un emploi sûr, stable et avec des droits)".

Ensuite, du point de vue du contenu signifié, elle a deux objets privilégiés de référence. D'une part, la forte flexibilisation du marché du travail en 1984 et 1992/94 par la régulation législative, dont l'objet principal a été la «normalisation» des contrats de travail «temporaires». Les syndicats se réfèrent ensuite au taux élevé de contrats de travail «temporaires » (entre 30 et $35 \%$ de l'ensemble des contrats), qui se maintient sans changements significatifs depuis la fin des années 1980. Ceci fait que, dans le discours syndical, «précarité» et caractère temporaire des contrats de travail et de l'emploi ont un rapport à la fois étroit et ambigu. Étroit, parce que le caractère temporaire des contrats est conçu comme la principale expression et cause de la "précarité», jusqu'au point de les assimiler parfois (8). Ambigu, parce que, assez fréquemment, les deux termes sont utilisés l'un à côté de l'autre, suggérant ainsi que si le caractère temporaire fait toujours partie de la précarité et lui est central, celle-ci a une signification qui va au-delà de la première. Cet «au-delà» a été explicité, par exemple, par un des membres du Comité exécutif de CCOO dans les termes suivants: "(Même si) en Espagne, les conditions d'emploi précaires (sont) identifiées fondamentalement avec le fait qu' un tiers du salariat a un emploi "temporaire" [...], derrière le référent de la précarité du travail, on trouve diverses situations concrètes qui ont à voir, premièrement, avec le chômage, mais aussi avec les formes d' "être" et d'accès à l'emploi, en définitive, avec les conditions d'emploi identifiées par un taux élevé d'emploi "temporaire" et de rotation dans les emplois, avec l'emploi à temps partiel, avec l'emploi souterrain.

(6) On peut consulter aussi les revues de Sociología del Trabajo et Cuadernos de Relaciones Laborales.

(7) Voir les revues Union de UGT et Gaceta sindical de CCOO.

(8) Le manifeste intersyndical du Premier Mai 2007 revendiquait «une réduction du taux de précarité de l'emploi» l'identifiant à une réduction du taux de contrats temporaires. 
Ce sont des conditions d'emploi qui déterminent en grande partie les conditions de travail spécifiques, comme l'insécurité généralisée, les bas salaires, le temps de travail non rétribué, l'indétermination et la disponibilité horaire exigée du travailleur, l'exécution de l'activité en situation de risque pour la santé» (Liceras Ruiz, 2000, p. 11). La précarité de l'emploi est ainsi vue par cette syndicaliste comme un phénomène aux contours diffus et multidimensionnel. Pour elle, les emplois précaires sont assimilés en Espagne, fondamentalement, à des emplois «temporaires». Cette identification est tellement forte et socialement puissante qu'elle est devenue aussi le signe distinctif de la définition scientifique espagnole. D'après l'un des meilleurs experts espagnols en économie de l'emploi, "employment precarisation, is a recurrent topic in academic and political discussion about Spanish Labour Market" The debate has focussed to explain the high rates of temporary employment (ReCIO, 2003, p. 1)(9).

Si on accepte comme point de départ cette définition de la précarité de l'emploi qui l'assimile aux emplois «temporaires», la question à se poser est d'abord celle des circonstances qui sont à l'origine de cette identification, pour essayer, ensuite, d'approfondir sa signification. Trois circonstances principales doivent être rappelées. Premièrement, le fait que l'opposition entre contrats de travail à durée indéterminée («fixes») et contrats «temporaires» va bien au-delà de leur acception. Elle doit se comprendre comme l'expression concentrée et symbolique des traits qui caractérisent deux normes sociales d'emploi historiquement différentes: celle qui «régulait» les rapports d'emploi jusqu'au milieu des années 1980, qu'on désigne habituellement par «norme salariale d'emploi», et celle qui les «régule» depuis les années 1990, qualifiée comme «norme flexible entrepreneuriale d'emploi » (PRIETO, 2002). Deuxièmement, le fait que les emplois «temporaires» concentrent les conditions d'emploi (et de travail) les plus mauvaises sur le marché du travail espagnol: instabilité involontaire dans l'emploi, bas salaires, accidents de travail, protection sociale faible, etc. Troisièmement, le fait que, dès le début des années 1990, les contrats «temporaires » touchent de façon permanente une partie très importante des salariés, soit directement par l'usage très étendu qui en est fait (30 à $35 \%$ des salariés concernés), soit indirectement du fait de la fragilisation générale du rapport salarial qui s'en suit.

(9) À vrai dire, cette sorte de réduction semble aussi assez fréquente dans la littérature européenne. Ainsi Düll, après un survey de littérature sur le sujet, conclut: "Although most of these dimensions (lower security of employment continuity, [...] lower pay and lower social benefits and pensions and possible worse working conditions) are mentioned in the literature reviewed, and although precarious employment is sometimes explicitly as a multidimensional phenomenon, the key criterion is that of employment contract (or absence of contract)" (DüLL, 2003a: 49).

\section{Le caractère temporaire des contrats comme précarité (1): un point critique de la norme sociale de l'emploi actuelle}

Les contrats «temporaires» sont à prendre comme l'expression condensée d'un changement profond dans la forme de régulation sociale de la relation salariale, que les syndicats ont refusé: ce refus a donné lieu entre 1988 et 2002 à quatre grèves générales d'une grande répercussion sociale. D'où l'intérêt d'étudier le passage du modèle précédent de régulation, qu'on caractérisera comme «norme salariale d'emploi», au modèle actuel, qu'on caractérisera comme «norme flexible entrepreneuriale» (pour un approfondissement, voir PRIETO, 2002 et 2006). Nous le ferons en suivant l'évolution des normes juridiques concernant l'emploi, tout en tenant compte du contexte de l'évolution de l'emploi et surtout du chômage rappelé dans le graphique 1 infra.

\section{Encadré 1 \\ Les matériaux utilisés}

Principales dispositions normatives concernant les relations d'emploi utilisées dans cet article pour l'étude de la précarité

- Loi 16/1976 des relations professionnelles, première loi de l'après-franquisme (et préconstitutionnelle) régulant les relations individuelles et collectives de travail.

- Constitution espagnole de 1978.

- Loi 8/1980 du statut des travailleurs, première version constitutionnelle de la loi organique régulant les relations individuelles et collectives de travail.

- Loi 51/1980 fondamentale d'emploi par laquelle on régule l'emploi et les institutions concernées.

- Loi 32/1984 sur modifications de certains articles de la loi 8/1980 concernant, surtout, les modalités du contrat de travail.

- Loi 22/1992 de mesures urgentes de promotion de l'emploi et de protection du chômage par lesquelles, entre autres, on rendu plus restrictive la perception des prestations par situation de chômage.

- Loi 11/1994 qui modifie certains articles du statut des travailleurs, dans un sens qui augmente le pouvoir entrepreneurial de gestion de l'emploi.

- Loi 63/1997 de mesures urgentes pour l'amélioration du marché du travail et la promotion des contrats à durée indéterminée.

- Loi 56/2003 d'emploi, actualisation de la loi $51 / 1980$.

-Décret-loi royal 5/2006 pour l'amélioration de la croissance et de l'emploi, orienté à promouvoir la stabilité dans l'emploi. 
Graphique 1: Évolution des taux d'activité, taux d'emploi et taux de chômage en Espagne (1976-2007)

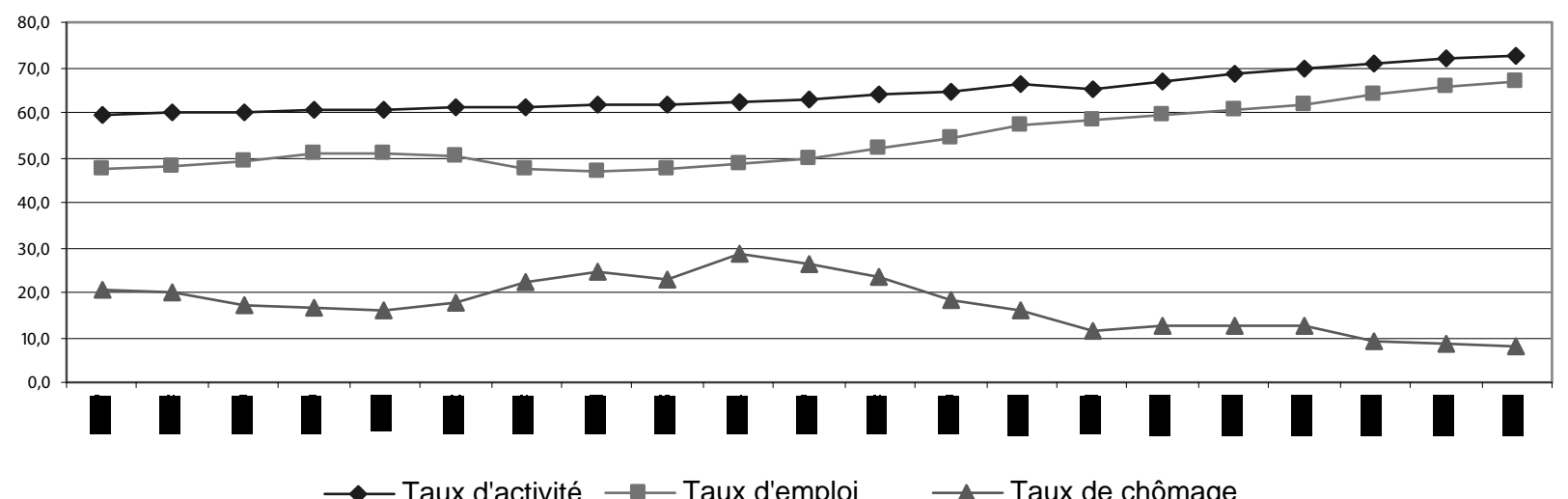

Source: Institut de la statistique espagnol (INE), enquête sur la population active (EPA). Les données correspondent au deuxième trimestre de chaque année.

Dans l'histoire démocratique récente de l'Espagne, il y a un moment où les traits de la norme salariale d'emploi précédente sont particulièrement manifestes. Ce sont les années où l'on a promulgué la loi sur les relations professionnelles (1976) et approuvé la Constitution espagnole actuelle (1978), le Estatuto de los Trabajadores (Statut des travailleurs) (1980) et la Ley Básica de Empleo (loi fondamentale d'emploi) (1980). Toutes ces lois sont l'expression et la concrétisation juridique d'une même conception de ce qu'est et doit être l'emploi - c'est-à-dire, une même norme sociale d'emploi - dans la société démocratique telle que la concevait la société espagnole au moment de la transition politique. Deux traits principaux vont caractériser cette norme: d'un côté, le «plein emploi» comme objectif politique général et la protection des travailleurs en cas de chômage involontaire (Ley Básica de Empleo, 1980); de l'autre, le pari en faveur d'un emploi stable et à temps complet, un «bon emploi» (Estatuto de los Trabajadores, 1980). «Plein emploi» et «bon emploi», les deux traits devaient aller ensemble: ni «plein emploi » sans «bon emploi », ni «bon emploi» sans «plein emploi».

Le «bon emploi» était donc conçu, avant tout, comme un emploi à forte stabilité, nommé emploi fixe dans la terminologie de l'époque venant de la période franquiste. La «fixité» était constatable empiriquement: à la fin des années 1970, 88 \% de travailleurs avaient une ancienneté dans leur entreprise de plus de cinq ans et $64 \%$ de plus de dix ans (PRIES, 1988). Mais, si on pouvait la constater empiriquement, c'était parce que la stabilité de l'emploi faisait partie de la norme sociale juridiquement établie. D'après la loi, il n'y avait qu'un type de contrat normal: le contrat à durée indéterminé (le «contrat fixe»). Par principe et par norme, tous les contrats de travail étaient présumés avoir une

Encadré 2
L'enquête sur la population active
(EPA)
«L'EPA est une enquête continue et de pério-
dicité trimestrielle auprès des familles, réalisée
par l'Institut national de statistique espagnol
(INE) depuis 1964. Sa finalité principale est
d'obtenir des données sur la force de travail et
ses différentes catégories (occupés, chômeurs,
etc. ), de même que sur la population inactive.
L'échantillon initial est de 65000 familles par
trimestre, ramené dans la pratique à environ
60000 familles interviewées de manière effec-
tive, ce qui correspond à 200000 personnes"
(INE). La question concernant le type de contrat
est posée seulement aux salariés occupés et
dans les termes suivants: "Votre contrat ou
relation de travail, est-elle de durée indéter-
minée ou limitée? ».

durée indéterminée, à moins que la durée de l'objet du contrat ne fût objective et précise (10). C'était la règle et la pratique. Le trait le plus significatif à ce propos venait des conditions requises pour la résiliation du contrat à l'initiative de l'entreprise. Le licenciement individuel du travailleur n'était en aucun cas discrétionnaire: il devait être justifié soit par des causes «objectives», que la loi limitait et précisait, soit pour des raisons disciplinaires, que

(10) Le Statut de travailleurs admettait quatre types de contrats limités dans le temps: contrat pour la réalisation d'une œuvre ou service concrets, en raison d'une forte demande (éventuel), intérimaire et de formation. Il va sans dire que la fin de ces contrats ne posait pas le moindre problème aux entreprises. 
l'entreprise devait pouvoir défendre ou prouver devant la magistrature du travail. Le licenciement «objectif» donnait droit, pour le travailleur, à une indemnisation de vingt jours de salaire par année travaillée jusqu'à un maximum de douze mois. Le licenciement «objectif » (ou «disciplinaire ») déclaré abusif obligeait l'entreprise à payer au travailleur une indemnisation de quarante-cinq jours de salaire par année travaillée jusqu'à une limite de quarantedeux mensualités, plus le montant des salaires non perçus par le salarié entre la date de communication du licenciement et celle de la sentence. Les limites établies pour les licenciements collectifs étaient d'un ordre différent. Dans ce cas s'ouvrait un processus formel de régulation d'emploi qui devait commencer par une négociation avec les représentants des travailleurs (le comité d'entreprise (11)) et devait se conclure par une résolution de l'administration publique du travail. En cas de résolution positive, les travailleurs affectés avaient droit, au minimum, à une indemnisation d'au moins vingt jours de salaire par année travaillée jusqu'à un maximum de douze mensualités.

Toutes ces conditions imposées à l'entreprise pour limiter son pouvoir discrétionnaire vis-à-vis de l'extinction du contrat de travail à durée indéterminée favorisaient la forte stabilité de l'emploi. La conjonction entre la stabilité de l'emploi et le pleinemploi (bas taux de chômage) faisait du rapport à l'emploi un rapport sûr pour tous les travailleurs ou presque. Mais le pari pour une stabilité à l'emploi particulièrement forte n'était pas la seule dimension spécifique de la norme salariale espagnole de l'emploi des années de la Constitution. Il y en a une deuxième tout aussi importante puisqu'il s'agit du type d'emploi qu'on voulait stabiliser. Le bon emploi devait être aussi à temps complet. Quand le Statut des travailleurs de 1980 inclut le temps partiel comme une des modalités possibles d'embauche, il le fait avec beaucoup de précaution: elle ne pourra être utilisée que d'une façon très limitée, seulement pour embaucher des bénéficiaires de l'assurance chômage et des jeunes de moins de 25 ans et ceci comme mesure de promotion de leur emploi.

L'option forte en faveur d'un emploi à temps complet et stable (fixe) fut donc la façon pour l'Espagne d'établir la norme salariale d'emploi. Temps complet et stabilité de l'emploi étaient ainsi conçues comme des pièces clés d'un ordre social où l'activité de travail (salarié) et la figure $\mathrm{du}$ travailleur occupaient/devaient occuper une place centrale. Comme norme, un vrai travail et un emploi authentique ne pouvaient pas être à temps partiel et/ou à durée déterminée. On considérait que ces types d'emploi impliquaient en soi un jugement

(11) Dans le droit de travail espagnol, le comité d'entreprise est constitué exclusivement par des représentants de travailleurs élus dans les centres de travail. moral social qui dégradait tant le travail que le travailleur embauché. L'emploi «temporaire» n'offrait qu'insécurité économique, l'emploi à temps partiel qu'insuffisance économique, l'emploi de caractère temporaire et à temps partiel qu'insécurité et insuffisance. Or, ni l'insécurité ni l'insuffisance économiques dues à l'emploi ne pouvaient être des propriétés sur lesquelles construire un ordre de relation salariale démocratiquement acceptable. «Par norme, tout emploi devait offrir des conditions minimes de sécurité et suffisance. Par norme, tout travailleur, disposant de capacités physiques, intellectuelles et morales de travail, devait avoir un emploi sûr et suffisamment rémunéré. Par norme, le fait d'avoir un emploi éliminait/devait éliminer toute possibilité d'être pauvre ou socialement exclu. La pauvreté et l'exclusion sociale n'étaient concevables qu'en dehors de l'espace social de l'emploi » (Prieto, 2002, p. 96). La sécurité et la suffisance se prolongeaient dans la protection du chômage et, à long terme, dans la protection de la retraite; dans le cas de la première, conçue en termes exclusivement contributifs, le travailleur touchait une allocation qui pouvait durer jusqu'à dix-huit mois avec un taux de substitution atteignant $80 \%$ du salaire précédant. En Espagne, la protection socio-économique du chômeur n'a jamais atteint le niveau accordé par d'autres pays européens avancés, mais c'est dans ces années, les années de la Constitution, qu'il a été le plus élevé. Au total, les emplois à caractère temporaire et à temps partiel étaient à ce point hors de la norme qu'ils n'étaient même pas intégrés dans les statistiques officielles d'emploi(12).

Tout cela prit place dans les institutions espagnoles à la fin des années 1970 et au début des années 1980, malgré la forte progression du chômage à ce moment-là $(4,8 \%$ en $1977,11,1 \%$ en 1980$)$. Les choses vont pourtant changer très vite. La perte d'emplois sera tellement importante et accélérée qu'en 1984 le taux de chômage atteindra 20\% de la population active (voir graphique 1 supra). Les mille petites mesures prises en faveur de l'emploi des «catégories défavorisées» ne réussissent pas à arrêter la saignée. En même temps, un vent de «flexibilisation» de l'emploi commence à bousculer toutes les institutions de la norme salariale de l'emploi des pays à économie avancée, qui commencent à devenir «rigides» (BOYER, 1986; RAPPORT DAHRENDORF, 1986). La flexibilisation va se mettre en place en Espagne sur le plan normatif en deux grandes secousses. La première a lieu en 1984 et la deuxième en 1992-1994, précédée, comme la précédente, d'une très forte augmentation du chômage: le taux de chômage atteint en 1994 le plus haut

(12) Le contraste sur ce point avec d'autres pays européens est clair. Les statistiques de l'emploi élaborées par la Commission européenne fournissent des données sur le temps partiel du début des années 1980 dans tous les pays de la Union européenne à quinze, sauf l'Espagne. 
niveau de toute l'histoire de l'emploi sur laquelle on dispose d'informations statistiques en Espagne, soit $24,5 \%$ des actifs.

La première grande modification de la norme sociale de l'emploi va donc se faire en 1984. La voie choisie sera la réforme du Statut des travailleurs par la loi 32/1984. Celle-ci conservera sans changer une virgule les règles concernant le contrat à durée indéterminée (en particulier, celles du licenciement), mais va libérer de toute entrave l'utilisation d'une modalité de contrat à durée limitée sans cause, sous la dénomination de "contrat "temporaires" comme mesure de promotion de l'emploi » : ce contrat avait été approuvé en 1980 pour faciliter les embauches aux entreprises, mais son usage était soumis à de très fortes restrictions. Dorénavant, ce sera une modalité contractuelle que les entreprises pourront utiliser sans aucune limite. N'importe quelle entreprise pouvait embaucher des travailleurs de toute qualification et en tout nombre pour une durée minimum de trois mois et maximum de trois ans; son extinction au terme de la période accordée ne comportait, de plus, aucun frais d'indemnisation. Ce type de contrat «est approuvé sans date de caducité, devenant ainsi un élément structurel du système juridique d'embauche» (TOHARIA, 2005: 27 (13)). Si l'option en faveur de la stabilité contractuelle avait été forte, avec la nouvelle modalité d'embauche temporaire, l'option en faveur d'une flexibilité contractuelle est tout aussi forte. Les facilités et les avantages de toutes sortes qu' elle offrait aux entreprises par rapport à tous les autres types de contrat vont faire d'elle la formule idéale d'embauche et de gestion entrepreneuriale du rapport d'emploi. En peu de temps et coïncidant avec une importante reprise de l'emploi, le nombre de travailleurs avec un contrat «temporaire» va augmenter très au-dessus de toute expectative: s'ils ne représentaient en 1984 que 7,6\% des salariés occupés (14), en 1987 le pourcentage sera de $15,3 \%$, et en 1991 , de 32,2\% (données EPA; voir graphique 2 infra). En sept ans, le chiffre d'emplois «temporaires» est ainsi multiplié par quatre ! Les contrats «temporaires» s'installent de cette façon massivement sur la scène du marché du travail espagnol.

L'extrême facilité offerte aux entreprises d'utiliser ces contrats «temporaires»- et, donc, leur normalisation - n'est pas le seul changement du moment. Il y en a un autre qui, à notre avis, a une signification sociale tout aussi importante, même si son incidence quantitative sera inférieure: le temps partiel change de statut. Dorénavant, le contrat de travail à temps partiel sera une modalité d'embauche aussi normale que le contrat à temps complet. En termes

(13) Cette œuvre sur le "problème des contrats "temporaires" en Espagne», dirigée par Toharia est, sans doute, la meilleure approche scientifique existant en Espagne sur la question.

(14) L'EPA ne commence à offrir des données statistiques différenciées par types de contrat qu'en 1987. Le chiffre de 1984 est une estimation de Montoya Melgar (1999). de normativité juridique, il n'y aura plus aucune différence entre les deux. Cependant, en contraste avec l'embauche temporaire, les entreprises s'en serviront beaucoup moins et presque toujours sous la modalité de contrat «temporaire » (face au 32,2\% du taux de contrat à durée limitée en 1991, celui de temps partiel ne sera que de 4,4\%); au bout de quelques années apparaîtra une exception sur ce point: celle de l'emploi des femmes. La nouvelle modalité d'embauche, le contrat «temporaire» comme mesure de promotion de l'emploi», sera donc la grande voie suivie par les entreprises pour flexibiliser leur usage de la force de travail. Quant à la protection sociale des chômeurs, sous la pression des syndicats, la loi 31/1984 va améliorer les conditions de perception de l'assurance-chômage, mais, en même temps, va ouvrir une nouvelle voie de protection qui entraînera des conséquences importantes, puisqu'elle sera une protection à caractère non contributif mais assistanciel.

En tout cas, la démarche flexibilisatrice de l'emploi venait de faire le pas le plus important: au moins sur le plan juridique, les quatre piliers centraux de la norme salariale de l'emploi espagnole précédente, c'est-à-dire, le plein-emploi, la stabilité - «fixité»-dans l'emploi (par la voie de la prévalence des contrats à durée indéterminée), la prestation du travail pendant des journées pleines (contrats à temps complet) et une assurance-chômage fermée à toute mesure protectrice à caractère assistancielle, étaient remis en cause. Avec eux, c'était aussi la fin de la norme salariale en tant que telle.

Une fois la boîte de la norme salariale d'emploi ouverte, la voie vers une flexibilisation beaucoup plus étendue était ouverte. Une première preuve en sera fournie par la tentative du gouvernement de prendre, en 1988, des mesures de flexibilisation pour l'embauche de jeunes, qui devaient théoriquement améliorer leur situation sur le marché du travail. Mais ceci restera au stade de l'essai du fait d'une grève générale qui paralysera le pays et obligera le gouvernement à faire marche en arrière. Cet «échec» sera, pourtant, suivi de deux autres lois qui atteindront leur objectif. La première sera le décret-loi 1/1992, dont l'objet consistera en «la rationalisation de la dépense en protection du chômage », une rationalisation qui comportera une réduction du niveau de protection. $\mathrm{Si}$, auparavant, la durée de l'assurance-chômage était la moitié de la période cotisée, elle ne sera plus désormais que d'un tiers, le maximum de durée étant fixé à deux ans. Et si, auparavant, le montant de la perception contributive était de $80 \%$ du salaire pendant les six premiers mois, elle ne sera plus désormais que de $70 \%$ et se verra réduite à $60 \%$ une fois passés six mois. La maigre protection des travailleurs en situation de chômage dans un marché de travail où le taux de chômage a toujours été très élevé - le nombre des chômeurs qui ont droit à une prestation contributive n'atteignant en moyenne les cinq dernières années que $31,1 \%$ du total - sera une circonstance qui va 
affaiblir substantiellement et de façon constante la position des salariés en contrat «temporaire» vis-àvis des entreprises.

La deuxième loi sera la loi 11/1994, qui va modifier profondément le Statut des travailleurs de 1980 en adaptant son contenu normatif aux besoins de la «nouvelle économie». Si la version du Statut de travailleurs de 1980 est l'expression et la concrétisation sur le plan juridique de la norme salariale d'emploi, celle de la loi 11/1994 sera l'expression et la concrétisation juridique de la norme d'emploi «flexible entrepreneuriale». Cette norme est l'expression d'une nouvelle doxa qui change l'ordre des priorités politiques: si, auparavant, c'était l'entreprise qui devait s'adapter aux exigences du (bon) emploi, désormais c'est l'emploi qui devra s'adapter aux besoins des entreprises. Du point de vue de la politique économique, c'est le passage de la politique de demande à la politique de l'offre (BILBAO, 1999). Un paragraphe de l'exposé de motifs nous met sur la piste: "On ne peut pas ignorer que le retour de la croissance économique et l'amélioration de la compétitivité des entreprises, en tant que pilier indispensable du maintien et de la croissance de l'emploi, requièrent, en plus de mesures économiques, qu'on entreprenne la réforme du cadre institutionnel de la relation d'emploi, afin de [...] permettre [...] l'adaptabilité aux changements des processus productifs et aux innovations technologiques». Approuvée au cœur de la crise de l'emploi la plus grave des temps démocratiques $(24,6 \%$ de chômage, $34,3 \%$ de contrats courts)(15), la loi $11 / 1994$ cherche une issue en accordant aux entreprises un pouvoir de contrôle de la gestion de la force de travail beaucoup plus étendu que celui qu'elles avaient auparavant, visant surtout la flexibilité interne mais aussi, secondairement, externe (16). Les syndicats ont réagi vigoureusement contre les deux lois, au point de provoquer contre elles deux grèves générales, l'une le 22 mai 1992 contre la première loi et l'autre le 27 janvier 1994 contre la deuxième.

(15) Malgré les prédictions des experts, la chute de l'emploi dans les années 1991-1994 va affecter beaucoup plus les emplois salariés fixes, ceux qui supposaient pour les entreprises un coût élevé d'indemnisation des travailleurs licenciés, que les emplois courts, ceux pour lesquels un terme mis à la relation d'emploi ne coûtait rien. Cette évolution pointait déjà l'option des entreprises en faveur d'une relation «souple» avec leurs employés.

(16) La loi accorde maintenant aux entreprises le pouvoir de «décider, par des raisons économiques, techniques, organisationnelles ou de production, des modifications substantielles des conditions de travail (comme) journée, horaire, travail posté, système de rémunération et système de travail» si ces modifications affectent moins de dix travailleurs dans les entreprises de moins de cent salariés, moins de $10 \%$ dans les entreprises de cent à trois cents salariés et moins de trente travailleurs dans les autres, sans avoir à les négocier, comme c'était le cas jusqu'ici, avec les représentants des salariés (article 5, trois). Concernant la flexibilisation externe, la loi légalise les entreprises d'emploi intérimaire.
Une fois établies sur le plan juridique les nouvelles règles de gestion interne de l'emploi, le problème de la durée des contrats deviendra le premier grand problème social à résoudre, pour les syndicats comme pour le gouvernement. Celui-ci, conscient du poids excessif des durées de contrat existantes, prend la décision de limiter fortement l'utilisation $\mathrm{du}$ «contrat temporaire» comme mesure de promotion de l'emploi», qui était de loin le plus utilisé dans les embauches temporaires; désormais, il ne pourra être utilisé sans limites, mais seulement dans des cas très précis et limités. La mesure n'aura, cependant, aucun résultat effectif sur la diminution des embauches temporaires (les entreprises se serviront d'autres modalités d'emploi «temporaire») mais, en même temps, elle ne sera que la première de toute une longue série. Suivront beaucoup d'autres: l' "Accord interconfédéral pour la stabilité de l'emploi» de 1997, qui aura comme suite la création d'une modalité de contrat à durée indéterminée avec un coût de résiliation plus bas; puis l'augmentation de la taxe sur les contrats courts de la loi 12/2001; plusieurs «plans extraordinaires» de stimulation économique à la reconversion par les entreprises de contrats courts en contrats à durée déterminée; la politique permanente des syndicats d'essayer d'atteindre dans la négociation collective des compromis en faveur de la stabilisation de l'emploi (Escudero RodríGuez, 2004); et enfin, toujours actuelle, la réforme et les nouvelles mesures législatives prises en faveur de la «stabilité contractuelle», suite à l' "Accord pour l'amélioration de la croissance et de l'emploi» signé entre les gouvernements et les partenaires sociaux le 9 mai 2006 et converti en norme publique par décret-loi 5/2006 le 9 juin.

Il serait faux de dire que le caractère temporaire de l'emploi soit le seul point de l'agenda sociopolitique actuel en Espagne: en 2002, il y eut une grève générale contre le projet gouvernemental de réforme de la protection des chômeurs qui renforçait la flexibilisation. Dans la mise en œuvre d'une nouvelle norme sociale d'emploi, la question prend toujours sur le plan social un caractère multidimensionnel. Mais l'évolution des relations entre acteurs sociaux montre que, dans les faits, c'est le caractère temporaire qui est le cœur de la nouvelle norme sociale: c'est en grande partie cette centralité qui la convertit en précarité.

Cette centralité n'est compréhensible, pourtant, que si l'on tient compte des deux autres circonstances signalées plus haut et qui vont être développées maintenant: d'un côté, l'étroit rapport entre les contrats «temporaires» et les conditions d'emploi et de travail les plus mauvaises et, de l'autre, la permanence dans le temps d'une précarité contractuelle qui touche directement une grande partie de la population salariée. 
Le caractère temporaire des contrats comme précarité (2): un rapport étroit avec de mauvaises conditions d'emploi et de travail

L'étroite coïncidence entre ces contrats «temporaires» et conditions d'emploi considérées socialement comme mauvaises, «indécentes» ou «indignes» contribue fortement à l'assimilation entre contrats «temporaires» et précarité. Cette coïncidence est souvent interprétée - particulièrement par les syndicats - en termes soit de causalité (la situation d'emploi temporaire amène à, ou porte en soi, d'autres conditions d'emploi précaires), soit de circularité (les unes vont presque toujours avec les autres).

Les conditions d'emploi habituellement associées à des contrats «temporaires » et considérées comme mauvaises ou de faible qualité sont les suivantes: le degré d'instabilité involontaire dans l'emploi, les salaires perçus, la faible protection sociale en situation de chômage et le taux d'accidents s du travail.

Tout d'abord, les salariés ayant un contrat de travail précaire subissent une instabilité dans l'emploi beaucoup plus élevée que ceux qui ont un contrat à durée indéterminée. Deux indicateurs appuient cette idée. Le premier est celui du passage par des situations d'emploi instables que subissent les salariés: au moins à court et moyen terme, une situation initiale d'emploi «temporaire» est suivie par des situations de chômage, de maintien dans des contrats instables ou même d'inactivité dans des proportions beaucoup plus élevées que si la situation initiale est celle de la stabilité (voir tableau 1). Le second indicateur concerne «l'ancienneté dans l'emploi actuel», qui est beaucoup plus réduite parmi les travailleurs «temporaires» que parmi les fixes: seulement 9,2\% des salariés en contrat «temporaires » ont une durée dans leur emploi actuel supérieure à six ans; par contre, ce pourcentage est de $58,2 \%$ parmi ceux qui ont un contrat à durée indéterminée (voir tableau 1).

Tableau 1: Degré d'instabilité dans l'emploi par type de contrats

\begin{tabular}{|l|c|c|c|c|c|}
\hline \multirow{2}{*}{$\begin{array}{c}\text { Type d'emploi } \\
\text { d'après la } \\
\text { modalité } \\
\text { contractuelle }\end{array}$} & \multicolumn{2}{|c|}{$\begin{array}{c}\text { Situation d'emploi une année } \\
\text { après la fin du contrat } \\
\text { (2001-2002) }\end{array}$} & \multicolumn{2}{c|}{$\begin{array}{c}\text { Durée dans } \\
\text { l'emploi actuel } \\
\text { (2005) }\end{array}$} \\
\cline { 2 - 6 } & $\begin{array}{c}\text { Contrats } \\
\text { à durée } \\
\text { indéter- } \\
\text { minée }\end{array}$ & $\begin{array}{c}\text { Contrats. } \\
\text { tempo- } \\
\text { raires }\end{array}$ & Chômage & $<\mathbf{1}$ an & $>$ 6 ans \\
\hline «Temporaire» & 14,5 & 73,0 & 12,5 & 56,7 & 9,2 \\
\hline $\begin{array}{l}\text { À durée } \\
\text { indéterminée }\end{array}$ & 93,4 & 4,4 & 2,2 & 7,2 & 58,2 \\
\hline
\end{tabular}

Source: Transitions d'emploi: enquête sur la population active, d'après les estimations de Laparra Navarro (2006: 167). Durée dans l'emploi: enquête sur la population active 2005, calcul propre. Pourcentages en ligne.

Non seulement la situation d'emploi des travailleurs «temporaires» est beaucoup plus instable, mais, leur rétribution salariale moyenne est assez nettement inférieure. Ainsi, d'après les données de la Encuesta de Estructura Salarial (enquête de structure salariale) de 2002 de l'Institut national de statistique, le salaire mensuel brut d'un travailleur «temporaires» $(1111,70$ euros $)$ ne représente en moyenne, que $60,7 \%$ de celui d'un travailleur fixe (1 840,70 euros).

Troisièmement, au-delà des conditions d'emploi, les conditions de travail des travailleurs «temporaires » se voient aussi négativement affectées si on les compare avec celles des travailleurs fixes. De tous les aspects les concernant, tant les syndicats que les experts prêtent attention prioritairement aux accidents de travail, ceux-ci comme un indicateur de la qualité de l'ensemble des conditions de travail. Il n'y a pas une seule étude qui traite du sujet et qui ne fasse pas remarquer le rapport étroit entre le type de contrat de travail et le nombre d'accidents(17). Ainsi le taux d'incidence générale des accidents de travail, calculé pour 2005 à partir des statistiques du ministère de Travail, est en moyenne 1,8 fois plus élevé parmi les travailleurs en contrat temporaire que parmi les travailleurs fixes. Et, d'après le «Rapport sur la santé au travail en Espagne. 2006», élaboré par l'Observatorio de Salud Laboral (2007), «(entre 1994 et 2004) les accidents mortels sont entre 2,5 et 3 fois plus élevés parmi les travailleurs en contrat «temporaire» que parmi ceux qui ont un contrat à durée indéterminée».

Quatrièmement, les travailleurs en contrat «temporaire» sont beaucoup moins protégés par rapport à la situation de chômage que ceux qui ont un contrat à durée indéterminée. Ceci ne s'explique pas seulement par le fait que la rupture de la relation d'emploi pour un contrat «temporaire» a un coût beaucoup plus bas pour l'entreprise (huit jours de salaire par année) que dans le cas d'un CDI (entre vingt et quarante-cinq jours par année). La période de cotisation exigée pour avoir droit à l'assurancechômage - qui doit dépasser une année - et la courte durée effective de leurs emplois font que leur indemnisation chômage sont ou bien inexistantes, ou bien d'une très courte durée. Cette condition de « chômeur sans droits» (ou quasiment) réduit leurs possibilités de trouver un bon emploi; d'après Laparra: «Un chômeur qui perçoit la prestation chômage voit ses risques d'accéder à un emploi "temporaire" réduits d'un tiers par rapport aux chômeurs qui ne touchent aucune prestation » (LAPARRA NAVARRO, 2006: 248).

Ces éléments, parmi beaucoup d'autres possibles, valident la thèse de la connexion entre contrats «temporaires» et mauvaises conditions d'emploi. On comprend que, dans ce contexte, un syndicat comme l'UGT puisse proposer dans un de ses

(17) Voir par exemple, Amuedo (2002), Hernanz, Toharia (2003) et, particulièrement, DuRÁN (2001). 
écrits revendicatifs récents: "Reducir la temporalidad para acabar con la accidentalidad" réduire le nombre de contrats de travail "temporaires" pour en finir avec les accidents du travail» (il aurait pu parler d'en finir avec l' «instabilité», les «bas salaires» ou la «protection inadéquate face au chômage»). $\mathrm{Au}$ moins pour les syndicats espagnols, dans les circonstances actuelles du marché du travail, l'amélioration de conditions de travail et d'emploi de faible qualité passe nécessairement par la réduction du nombre de contrats «temporaires», puisque c'est ce phénomène qui se trouve derrière.

\section{Le caractère temporaire des contrats comme précarité (3): la permanence d'un taux très élevé}

La position centrale du caractère temporaire des contrats dans la norme sociale actuelle de l'emploi espagnol décrite plus haut et le rapport étroit que l'on vient de signaler entre caractère temporaire des contrats et mauvaise qualité de diverses dimensions de l'emploi permettent de comprendre pourquoi, sous l'influence du mouvement syndical, la précarité des contrats est devenue le problème social clé des relations d'emploi. Mais ce n'est vrai qu'en partie: les contrats «temporaires» n'ont atteint en Espagne l'immense prégnance qui est la leur comme problème social et politique qu'à partir d'un troisième facteur: le taux très élevé des salariés qui sont touchés par cette forme de contrats et sa permanence dans le temps.

Son importance devient visible quand on compare ce même taux avec celui d'autres pays européens. Le taux des contrats «temporaires » n'est qu' un indicateur assez limité quand on veut analyser en termes comparatifs l'emploi «temporaire» dans plusieurs pays (ToHARIA, 2005: chapitre II); il reste très significatif que ce taux-là soit beaucoup plus élevé en Espagne que dans le reste des pays de l'Union européenne à quinze (voir tableau 2). La plupart des pays de l'Union européenne ont vu augmenter ces dernières années leur taux de contrats «temporaires»: pourtant, la différence entre le taux de contrats «temporaires» en Espagne et la moyenne de l'Union européenne à quinze en 2005 est toujours de 19 points d'après les données de l'Eurostat, soit plus du double. Une différence trop importante pour qu'elle n'ait pas de signification.

L'usage des contrats «temporaires » par les entreprises n'est devenue significatif qu'après la réforme législative du Estatuto des los Trabajadores de 1984, qui ouvre la voie à l'utilisation sans limites du «contrat «temporaire» comme mesure de promotion de l'emploi » (voir plus haut). Dès la mise en œuvre de la réforme, son usage est devenu massif en peu d'années, passant de 7,6\% du total de salariés occupés en 1984 à 15,3\% en 1987 et 32,2\% en 1992. Jusqu'à aujourd'hui, ce pourcentage s'est maintenu à peu près constamment (voir graphique 2). Depuis le début des années 1990 son taux a oscillé de $30 \%$ à $35 \%$. Il faut souligner que la permanence de ce taux sur plus de quinze ans a eu lieu dans des conjonctures économiques et de l'emploi très différentes: la forte chute de l'emploi du début des années 1990, accompagnée d'une importante augmentation du taux de chômage $(24,5 \%$ en 1994$)$; et la très forte croissance de la population active occupée depuis 1994 avec la réduction du chômage $(8,1 \%$ en 2006) (voir les articles de Toharia et Cebrián). Elle a eu lieu, aussi, malgré toutes les mesures publiques prises à partir de 1994 en faveur de la réduction de la précarité contractuelle et de l'augmentation des emplois stables. Le dernier pari public, et peut-être le plus fort, en faveur de la réduction de la précarité a eu lieu à l'occasion de l' "Accord pour l'amélioration de la croissance et l'emploi »; le taux d'emploi «temporaires» est descendu légèrement (à 31,94\% au troisième trimestre 2007), mais il faudra attendre pour avoir une perspective temporelle plus large. La constatation de la permanence dans le temps d'un même taux dans des circonstances différentes a conduit beaucoup d'experts à considérer qu'il est devenu un «trait structurel» du marché du travail espagnol (TOHARIA, 2005).

Autour d'un pourcentage moyen de $33,3 \%$ en 2005 (données annualisées de l'INE), il y a certaines catégories de salariés qui sont davantage touchés par les contrats «temporaires» que d'autres (voir

Tableau 2: Taux de contrats «temporaires» dans différents pays de l'Union européenne à quinze en \% de l'emploi total (2005)

\begin{tabular}{|c|c|c|c|c|c|c|}
\hline $\begin{array}{c}\text { Union européenne à } \\
\text { quinze }\end{array}$ & Espagne & Royaume-Uni & Allemagne & France & Italie & Suède \\
\hline 14,3 & 33,3 & 5,7 & 14,2 & 13,3 & 12,3 & 16,0 \\
\hline
\end{tabular}

Source: Eurostat, L'emploi en Europe, 2006.

Note: la classification statistique faite par Eurostat des contrats de travail d'après leur durée distingue entre: «Permanents jobs or work contract of in-limited duration» et toutes les autres modalités des contrats temporaires ensemble («temporary jobs/work contracts of limited duration») (DüLL, 2003: 11). 
Graphique 2: Évolution des taux d'emploi temporaire global, par sexe, groupes d'age et secteur (1988-2007)

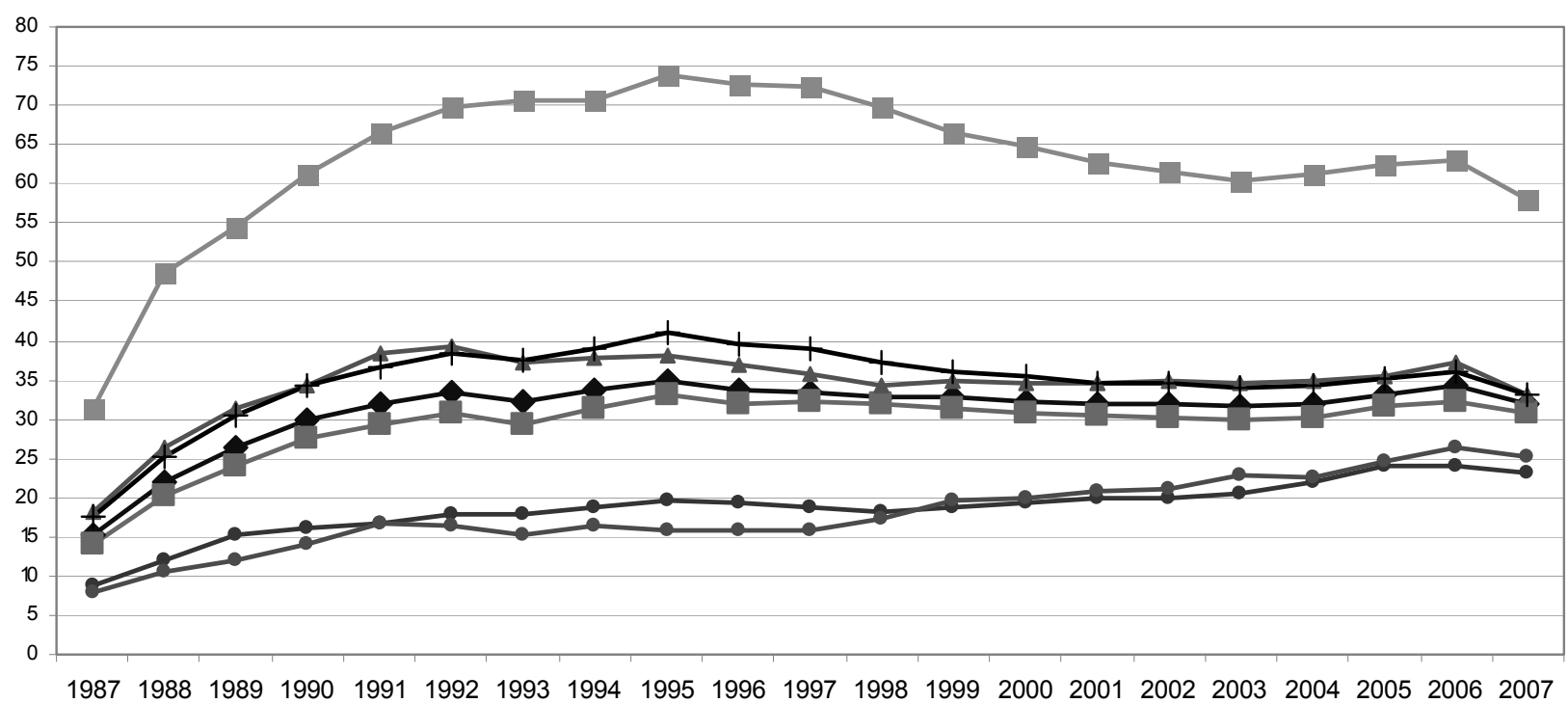

Total contrats temporaires $\rightarrow$ Hommes $\rightarrow$ Femmes $\longrightarrow 20$ à 24 ans

$\rightarrow 40$ à 49 ans

- Secteur public - Secteur privé

Source: Institut national de la statistique espagnol (INE), enquête sur la population active (EPA). Les données correspondent au deuxième trimestre de chaque année.

tableau 3). Toutes les situations, allant du genre à l'âge ou à l'origine nationale, sont discriminatoires par rapport au pourcentage de contrats «temporaires». Ces discriminations ont, d'ailleurs, un caractère presque structurel puisqu'elles changent peu au cours des années (voir graphique 2). Or, si on trouve des discriminations quel que soit le critère de classification des salariés, certaines variables sont à la source de discriminations quasi extrêmes : les travailleurs non-qualifiés, les non-diplômés, les immigrés et les très jeunes ont des taux de contrats «temporaires » qui, en 2005 , vont de $44 \%$ des nondiplômés et des non-qualifiés à $60 \%$ et $65 \%$ des très jeunes et des immigrés. Dans le cas de la différence par sexe, elle devient beaucoup plus conséquente si on tient compte - comme il le faut quand il s'agit du genre - non seulement des contrats à durée limitée mais aussi des temps partiels.
Il reste, sur ce point, à connaître les transitions contractuelles des personnes en contrat «temporaires » et surtout à savoir si, à moyen terme, elles parviennent à trouver la stabilité ou restent «bloquées» dans ce type de contrats, ou entre celui-ci et le chômage. Il n'existe malheureusement pas de données qui apportent une information directe et systématique sur cette question et très peu de recherches l'abordent d'une façon claire et précise (ToHARIA, CEBRIÁn, 2007). On peut prendre comme indicateur de tendance les résultats d'une étude réalisée par LAPARRA NAVARRo (2006). Se servant des registres administratifs du service public d'emploi de Navarre, il a réalisé un suivi des chômeurs embauchés sur des contrats «temporaires» en 1999 et étudié leur situation contractuelle cinq années après, en 2004. D' après ses calculs, sur la totalité des personnes embauchées sur des contrats «temporaires» en 1999, 41,5\% avaient un contrat à durée déterminée en 2004, mais $33 \%$ n'avaient eu que de contrats «temporaires», tandis

Tableau 3: Parts des contrats «temporaires» par variables en \% (2005)

\begin{tabular}{|c|c|c|c|c|c|c|c|c|c|c|c|}
\hline \multicolumn{2}{|c|}{ Genre } & \multicolumn{2}{|c|}{ Âge } & \multicolumn{2}{|c|}{ Origine } & \multicolumn{2}{|c|}{$\begin{array}{c}\text { Niveau de } \\
\text { formation** }\end{array}$} & \multicolumn{2}{|c|}{$\begin{array}{l}\text { Qualification } \\
\text { des postes** }\end{array}$} & \multicolumn{2}{|c|}{ Secteur } \\
\hline Femmes & Hommes & $16-24$ & 40-49 & Immigrés & Autochtones & $\begin{array}{c}\text { Sans } \\
\text { études }\end{array}$ & $\begin{array}{c}\text { Diplômes } \\
\text { universitaires. }\end{array}$ & $\begin{array}{c}\text { Manuels } \\
\text { non qualifiés }\end{array}$ & \begin{tabular}{|c|} 
Non manuels \\
qualifiés
\end{tabular} & Privé & Public \\
\hline $\begin{array}{c}35,5 \\
(48,0) *\end{array}$ & $\begin{array}{c}31,6 \\
(33,1)^{*}\end{array}$ & 65,3 & 24,1 & 60 & 31 & 44,0 & 19,5 & 44,0 & 20,0 & 35,2 & 24,7 \\
\hline
\end{tabular}

Source: enquête sur la population active (EPA) et traitement propre.

Lecture: les données des variables signalées avec le signe* et entre parenthèses sont des pourcentages dont la base est l'addition des contrats précaires et à temps partiel. Les données des variables signalées avec le signe** proviennent de Luis ToHARIA (2005) et sont de 2004. 
que $25,5 \%$ avaient alterné contrats «temporaires » et périodes de chômage. Autour de ces moyennes générales, on constate une distribution qui, du point de vue des transitions contractuelles, répond à la logique de diversification catégorielle qu'on vient de voir (voir tableau 4).

Tableau 4 : Transitions contractuelles des travailleurs qui ont eu emploi «temporaires» en 1999 : évolution jusqu'à 2004

\begin{tabular}{|c|c|c|c|}
\hline Positions\Transitions & 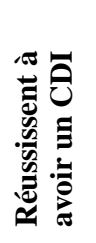 & 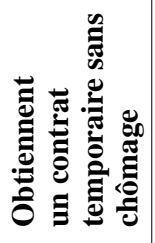 & 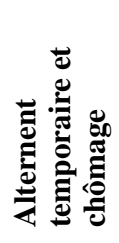 \\
\hline Hommes & 39,9 & 36,3 & 23,8 \\
\hline Femmes & 43,7 & 28,3 & 27,7 \\
\hline Sans études & 31,2 & 63,2 & 5,7 \\
\hline Études moyennes & 42,3 & 34,7 & 23,0 \\
\hline $\begin{array}{l}\text { Formation } \\
\text { professionnelle }\end{array}$ & 51,3 & 24,5 & 24,2 \\
\hline Universitaires & 42,5 & 28,5 & 29,0 \\
\hline Cadres & 43,2 & 35,9 & 21,8 \\
\hline Qualifiés non-cadres & 44,0 & 30,8 & 25,2 \\
\hline Non qualifiés & 38,8 & 34,4 & 26,8 \\
\hline Secteur industriel & 48,7 & 29,5 & 21,8 \\
\hline Bâtiment & 37,2 & 42,3 & 30,5 \\
\hline Services & 42,3 & 31,7 & 26,1 \\
\hline $16-29$ ans & 43,7 & 33,4 & 23,0 \\
\hline $30-44$ ans & 41,2 & 30,7 & 28,2 \\
\hline$>45$ & 33,5 & 37,2 & 29,0 \\
\hline Total & 41,5 & 33,0 & 25,5 \\
\hline
\end{tabular}

Source: Laparra Navarro, 2006: 178.

Note: les données se rapportent aux travailleurs qui ont eu un CDD en 1999 d'après les registres administratifs du service public d'emploi de Navarre (Espagne).

Pourtant, s'il est important de savoir quelles sont les catégories des travailleurs les plus touchées par l'emploi «temporaire » » et quelles sont leurs transitions vers des contrats stables, il l'est tout autant de constater qu'il n'y a pas une seule catégorie sociale qui y échappe de manière significative. Les hommes (face aux femmes), les adultes (face aux jeunes), les travailleurs autochtones (face aux immigrés), les diplômés de l'enseignement supérieur (face aux non diplômés), ceux qui occupent un poste qualifié (face à ceux qui occupent un poste non-qualifié), les employés du secteur public (face à ceux du secteur privé), toutes ces catégories de salariés ont des taux de contrats «temporaires» nettement moins élevés que leurs contraires, mais jamais à un taux inférieur à 19,5\% (celui des diplômés de l'enseignement supérieur; voir tableau 3). Ceci veut dire que cette question et tout ce qu'elle implique sur le plan social atteint la quasi-totalité de la population salariée, quelle que soit sa position sur le marché du travail, que ce soit directement ou comme une éventualité plus ou moins rapprochée.

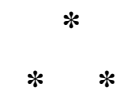

Les éléments que nous venons de parcourir explicitent les raisons de l'assimilation qui existe en Espagne entre contrats «temporaires» et précarité de l'emploi comme problème social; ils permettent aussi d'approfondir le contenu de la notion même de précarité. C'est en soulignant ce contenu et en faisant voir sa particularité que l'on veut terminer cet article.

La définition de la précarité de l'emploi se déploie en Espagne sous quatre aspects ou attributs, tous nécessaires pour en comprendre la spécificité qui résulte justement de leur combinaison et de leur interaction.

Le premier de tous tient précisément à la normalisation, juridique et pratique, de l'usage des contrats de travail «temporaires», une normalisation qui fait partie de la norme sociale de l'emploi actuellement hégémonique et qui n'est pas considérée - du moins pour les syndicats - comme adéquate à ce que devrait être le rapport d'emploi dans une société démocratique.

Le caractère temporaire de l'emploi est ainsi le premier des attributs qui définissent la précarité, celui qui occupe le devant de la scène, mais il se voit habituellement accompagné par d'autres conditions d'emploi et de travail dont la qualité est, elle aussi, au-dessous de leur devoir-être socialement reconnu: haute instabilité involontaire, bas salaires, faible protection sociale, mauvaises conditions de travail (longues journées, autoritarisme, forte intensité, horaires asociaux...) qui augmentent la probabilité d'accidents, la précarité est ainsi plus que «caractère temporaire»: un travailleur «temporaire» à mobilité volontaire, bien payé, grande autonomie, etc. ne serait pas précaire.

Un taux de contrats "temporaires» très élevé, - dans un contexte d'un niveau de chômage qui s'est étendu de $10 \%$ à $20 \%$ de la population active - qui, bien qu'il atteigne de manière spécifique certaines catégories des salariés, les concerne toutes de façon généralisée et de manière permanente au cours des années. Sans cet aspect le problème social de l'emploi à caractère temporaire et de la précarité de l'emploi aurait été très différent ou il n'aurait même pas existé.

Et finalement, mais ce n'est pas le moindre, le fait qu'il y ait une force sociale, comme le syndicalisme, très présente dans la société civile et d'une grande incidence politique, qui ait converti la question du caractère temporaire de l'emploi en point critique de la question sociale. À nouveau, sans cette implication du syndicalisme le problème social de l'emploi «temporaire», devenue précarité de l'emploi aurait été tout un autre ou n'aurait même pas existé. La précarité de l'emploi n'est pas une question dont 
l'existence dépend des analyses des chercheurs en sciences sociales.

La particularité du phénomène de la précarité de l'emploi en Espagne comme problème social ainsi que de sa définition, tant profane que scientifique, résulte de la combinaison des quatre éléments qu'on vient de résumer. On peut trouver, dans d'autres pays, des conceptions de la précarité de l'emploi par certains côtés semblables (ce serait en partie le cas de la France), mais, si on analyse le phénomène en profondeur, on verra qu'on ne trouve nulle part la même combinaison d'éléments ni, par conséquent, la même réalité ni la même définition. C'est, d'ailleurs, une façon d'être en harmonie avec un modèle d'État social qui combine une reconnaissance de droits sociaux très étendue avec des prestations économiques très faibles (voir l'article de Rodríguez Cabrero dans ce numéro) et avec un modèle de croissance économique et de compétitivité sur le plan international que certains chercheurs qualifient comme de Low Skill Equilibrium (EsPINGANDERSEn, 2000).

\section{Annexe}

\section{Norme salariale et norme flexible entrepreneuriale d'emploi en Espagne Traits généraux}

\begin{tabular}{|c|c|}
\hline Norme Salariale & Norme Flexible/Entrepreneuriale \\
\hline $\begin{array}{l}\text { I) Orientation politique globale et effets généraux } \\
\text { - Centralité sociale et politique de l'emploi (salarié) } \\
\text { - Plein emploi de «bon emploi» } \\
\text { - Taux de chômage réduit } \\
\text { - Expectatives parmi les salariés d'une amélioration constante } \\
\text { des conditions de travail et de vie }\end{array}$ & $\begin{array}{l}\text { I) Orientation politique globale et effets généraux } \\
\text { - Centralité de l'entreprise (compétitivité) } \\
\text { - Maximisation de l'emploi (ou «plein emploi » de tout sorte } \\
\text { d'emploi } \\
\text { - Taux de chômage élevé } \\
\text { - Incertitude généralisée }\end{array}$ \\
\hline $\begin{array}{l}\text { II) Régulation sociale des conditions d'emploi et de travail } \\
\text { - Contrat à durée indéterminée et à temps complet comme } \\
\text { norme unique } \\
\text { - Inexistence pratique des contrats temporaires et à temps partiel } \\
\text { - Salaires: suffisance et tendance à l'augmentation constante et } \\
\text { généralisée } \\
\text { - Pouvoir des entreprises vis-à-vis des conditions d'emploi : très } \\
\text { régulé }\end{array}$ & $\begin{array}{l}\text { II) Régulation sociale des conditions d'emploi et de travail } \\
\text { - Normalisation des contrats à durée déterminée et à temps } \\
\text { partiel } \\
\text {-Élevé taux de temporalité } \\
\text { - Salaires: grande diversité, variations incertaines } \\
\text { - Pouvoir des entreprises vis-à-vis des conditions d'emploi : } \\
\text { amplifié }\end{array}$ \\
\hline $\begin{array}{l}\text { III) Protection sociale du chômage } \\
\text { - Système contributif et de répartition } \\
\text { - Taux de substitution salariale élevée et périodes relativement } \\
\text { longues de prestations }\end{array}$ & $\begin{array}{l}\text { III) Protection sociale du chômage } \\
\text { - Combinaison du système contributif et de répartition et } \\
\text { assistenciel } \\
\text { - Taux de substitution salariale moindre et périodes moins } \\
\text { courts de prestations }\end{array}$ \\
\hline $\begin{array}{l}\text { IV) Sujets d'emploi de référence } \\
\text { - Hommes adultes } \\
\text { - Itinéraires d'emploi longs, stables, sûrs et généralisés } \\
\text {-Forte homogénéité de la classe salariée }\end{array}$ & $\begin{array}{l}\text { IV) Sujets d'emploi de référence } \\
\text { - Hommes adultes, mais aussi, à un niveau inférieur, femmes, } \\
\text { immigrés et jeunes } \\
\text { - Itinéraires d'emploi variables, incertains, épars, et } \\
\text { individualisés } \\
\text {-Classe salariée très segmentée }\end{array}$ \\
\hline
\end{tabular}

Source : élaboration propre à partir de PRIETO (2002). 


\section{Bibliographie}

Alonso L.E. (2000), Trabajo y posmodernidad: el empleo débil, Madrid, Fundamentos.

Amuedo C. (2002), "Work safety in the context of temporary employment: The Spanish Experience", Industrial and Labor Relations Review, 55 (2).

Bilbao A. (1999), El empleo precario. Seguridad de la economía e inseguridad del trabajo, Madrid, Libros de la Catarata.

BOyer R. (dir.) (1986), La flexibilidad del trabajo en Europa, Madrid, Ministerio de Trabajo y Seguridad Social.

CAno E. (2000), “Análisis de los procesos socioeconómicos de precarización laboral", in Cano E., Bilbao A. y Standing G. (2000), Precariedad laboral, flexibilidad y desregulación, Valencia, Germanía, pp. 25-68.

CARnoy M. (2001), El trabajo flexible en la era de la información, Madrid, alianza Editorial.

Castels M. (1999), La era de la información. Economía, sociedad y cultura, vol. 1, La sociedad red, Madrid, Alianza Editorial.

Cingolani P. (2007), Le travail précaire est-il nouveau? Esquisse d'une rétrospection des points de vue engagés, JIST.

Consejo Económico y Social (2006), España 2005. Memoria sobre la situación socioeconómica y laboral, Madrid, CES. Consejo Económico y Social.

DüLl N. (2003a), "Precarious Employment: Methodologies and Measurements. Analysis of precarious employment from a comparative perspective, by taking experiences of five European countries into account", Risk and Insecurity in Flexible Economies, European Framework 5 Programme. A seminar organised as part of the work of the ESOPE project (European Study of Precarious Employment), University of Warwick, 23 et 24 mai 2003, miméo.

DüLl N. (2003b), "Defining and assessing precarious employment in Europe: review of main studies and surveys", Risk and Insecurity in Flexible Economies. European Framework 5 Programme. A seminar organised as part of the work of the ESOPE project (European Study of Precarious Employment), University of Warwick, 23 et 24 mai 2003, mimeo.

DuRAN F. (2001), Informe sobre los riesgos laborales y su prevención, Madrid, Presidencia de Gobierno.

ESCUDERO RodRíGUEZ R., (coord.), (2004), La negociación colectiva en España: una mirada cualitativa, Valencia, CCOO, Tirant lo Blach.

EsPing-ANDERSEn G. (2000), Who harmed market regulation? in Esping-Andersen G. and Regini M., (ed.). (2000), Why De-Regulated Labour Markets? Oxford University Press.

Hernanz Martín V. (2003), El trabajo temporal y la segmentación. Un estudio de las transiciones laborales, Madrid, Consejo Económico y Social.

Herranz V., Toharia L. (2003), ¿Aumentan los contratos temporales la siniestralidad? Una comparación ente Italia y España, mimeo.

Liaisons Sociales magazine (2000), «L'Espagne veut perdre son titre de championne de l'emploi temporaire», novembre 2000, pp. 40-43.
LAPARRA NAVARRo M. (2006), La construcción del empleo precario, Madrid, Fundación FOESSA-Cáritas.

Liceras Ruiz D. (2000), "Precariedad laboral, políticas de empleo y representación sindical", in Cano E., Bilbao A., Standing G. (2000), Precariedad laboral, flexibilidad y desregulación, Valencia, Germanía, pp. 11-24.

MADSEN K. (2004), "The Danish model or "flexicurity": experiences and lessons", Transfer. European Review of Labour and Research, Vol. 10, $\mathrm{n}^{\mathrm{o}} 2$, summer 2004, pp. 187-207.

Miguélez F. (2004), "La flexibilidad laboral”, Trabajo. Revista de la Asociación Estatal de Centrso Universitarios de RR LL. y CCTT, no 13, janvier-juin, 2004.

Miguélez F., Prieto C. (ed.) (1991), Las relaciones laborales en España, Madrid, Siglo XXI.

Miguélez F., Prieto C.(ed.) (1999), Las relaciones de empleo en España, Madrid, Siglo XXI.

Montoya Melgar A. (1999), La estabilidad en el empleo: recuperación de un principio, Revista del Ministerio de Trabajo y Asuntos Sociales, $\mathrm{n}^{\circ} 33$, pp. 61-7.

Observatorio de Salud Laboral (2007), Informe de Salud Laboral España, 2006.

PRIES L. (1988), “Calificación, relaciones laborales y mercado de trabajo: el concepto de estrechez del ámbito empresarial", Revista Española de Investigaciones Sociológicas, $n^{\circ} 41 / 88$.

Prieto C. (ed.) (1999), La crisis del empleo en Europa, Valencia, Germanìa, 2 vols.

PRIETO C. (2002), "La degradación del empleo o la norma social del empleo flexibilizado", Sistema, no 168-169.

Prieto C. (2006), "The Degradation of Employment in Spain: From the Salaried Employment Norm to the Entrepreneurial Employment Norm", in Alonso L.E. y Martínez Lucio M. (2006), Employment Relations in a Changing Society. Assessing the Post-Fordist Paradigm, Londres, Palgrave, Macmillan, pp. 52-68.

RAPPORT DAHRENDORF (1986), OCDE, Paris.

Recio A. (2003), "Employment precarisation and labour market segmentation in Spain", Risk and Insecurity in Flexible Economies. European Framework 5 Programme. A seminar organised as part of the work of the ESOPE project (European Study of Precarious Employment), University of Warwick, 23 et 24 mai 2003, mimeo.

SENNetT R. (1998), La corrosión del carácter. Las consecuencias personales del trabajo en el nuevo capitalismo, Barcelona, Anagrama.

TOHARIA L. (dir.) (2005), El problema de la temporalidad en España: un diagnóstico, Madrid, Ministerio de Trabajo y Asuntos Sociales.

Toharia L., Cebrián I. (2007), La temporalidad en el empleo: atrapamiento y trayectorias, Madrid, Ministerio de Trabajo y Asuntos Sociales.

TuchsZiRer C. (2005), «Les différentes approches de la précarité de l'emploi en Europe, au Japon et aux ÉtatsUnis», Chronique internationale de l'IRES, $\mathrm{n}^{\circ} 97$, novembre 2005.

VALDÉs DAL-Ré F., LAHERA CORTEZA J. (2004), La precariedad laboral en España, Madrid, Comare. 